\title{
Neuraltherapie nach Huneke - das Phänomen der «Störfeldkette»
}

\author{
Dieter Thommen \\ Facharzt für Innere Medizin FMH / Neuraltherapie FMH/SANTH, CH-Bern
}

W enn bei einer neuraltherapeutischen Therapie die Wirkung einer lokalen oder segmentalen Behandlung unbefriedigend ist, muss an das Vorhandensein eines oder mehrerer Störfelder gedacht werden [1,2,3, 6]. Dabei handelt es sich um meist asymptomatische chronische Reizzustände, die prinzipiell überall im Körper vorkommen können. Die davon ausgehenden Reizimpulse können über eine Störung von Regulationssystemen (insbesondere über das sog. Grundregulationssystem [5,10] und den Sympathikus) prinzipiell jedes andere System des Organismus beeinflussen. Die neuraltherapeutische Ausschaltung des Störfeldes führt - falls dies anatomisch noch möglich ist - zur vollständigen Ausheilung der betreffenden Erkrankung; es handelt sich somit um eine kausal ausgerichtete Therapie.

Für den Patienten und den Therapeuten ist das Sekundenphänomen nach HunEKE $[7,8,9]$ ein äusserst eindrückliches Erlebnis, kommt jedoch nicht obligat bei jeder erfolgreichen Störfeldbehandlung vor. Das Sekundenphänomen ist folgendermassen definiert:

- Bei der Injektion von Procain oder Lidocain in bzw. an ein schuldiges Störfeld müssen sämtliche von ihm ausgelösten Fernstörungen in der gleichen Sekunde vollständig verschwinden (soweit dies anatomisch noch möglich ist).

- Dabei muss die Wirkung mindestens 20 Stunden (im Zahn-Kieferbereich mindestens 8 Stunden) anhalten.

Bei der neuraltherapeutischen Behandlung chronischer Krankheiten trifft man immer wieder auf das Vorhandensein mehrerer Störfelder. Am Beispiel von drei Patienten werden diese sogenannten Störfeldketten aufgezeigt und diskutiert. Störfeldketten weisen oft eine hierarchische Struktur auf, wobei die einzelnen Komponenten häufig chronologisch angeordnet sind. So kann sich im Behandlungsverlauf die Vorgeschichte des Patienten regelrecht rückwärts aufrollen, wie das Fallbeispiel 2 zeigt. Dabei spielen die sogenannten retrograden Phänomene oft eine entscheidende Rolle, da diese meist auf frühere Ereignisse hinweisen, die dem Patienten bei der Anamnese-Erhebung nicht mehr bewusst waren. Erst die konsequente neuraltherapeutische Behandlung sämtlicher Glieder einer Störfeldkette kann in solchen Fällen zum Erfolg führen.

Schlüsselwörter: Neuraltherapie nach Huneke, Störfeld, Störfeldkette

\begin{abstract}
Neural Therapy according to Huneke - The 'Chain of Interference Fields'
During neural therapy of chronic diseases, it is common to encounter several different interference fields. These so-called chains of interference fields are demonstrated and discussed, using three patients as examples. Chains of interference fields often exhibit a hierarchical structure, with a chronological alignment of the individual components. Thus the patient's prior history can really be unrolled backwards, as case 2 shows. This so-called "retrogrades Phänomen", i.e. a previously mute interference field which now becomes symptomatic, often plays a decisive role, as it indicates earlier events of which the patient was not aware when the history was taken. Consistent neural therapeutic treatment of all links in the chain of interference fields can bring success in such cases.
\end{abstract}

Key words: Neural Therapy according to Huneke, interference field, chain of interference fields

- Bei Wiederauftreten der Symptome muss das erneute Anspritzen des schuldigen Störfeldes denselben sofortigen Effekt haben und die Symptomfreiheit muss deutlich länger anhalten.

Nicht selten scheint die Ursache der Erkrankung nach einer erfolgreichen Störfeldtherapie gefunden $\mathrm{zu}$ sein. Nachdem anfänglich die Ausschaltung eines Störfeldes erfolgreich war, kann es aber unter Umständen zum Rückfall der Symptomatik kommen und dieselbe
Behandlung spricht im weiteren Verlauf nicht mehr an. In solchen Fällen liegt oft nicht nur ein einzelnes Störfeld, sondern eine Verkettung mehrerer Störfelder vor. Im Folgenden wird deshalb der Begriff "Störfeldkette" verwendet.

\section{Fallbeispiel 1}

Eine 40-jährige Patientin (Jahrgang 1963) wurde mir vom Zahnarzt zur Nachbehandlung zugewiesen. Wegen 


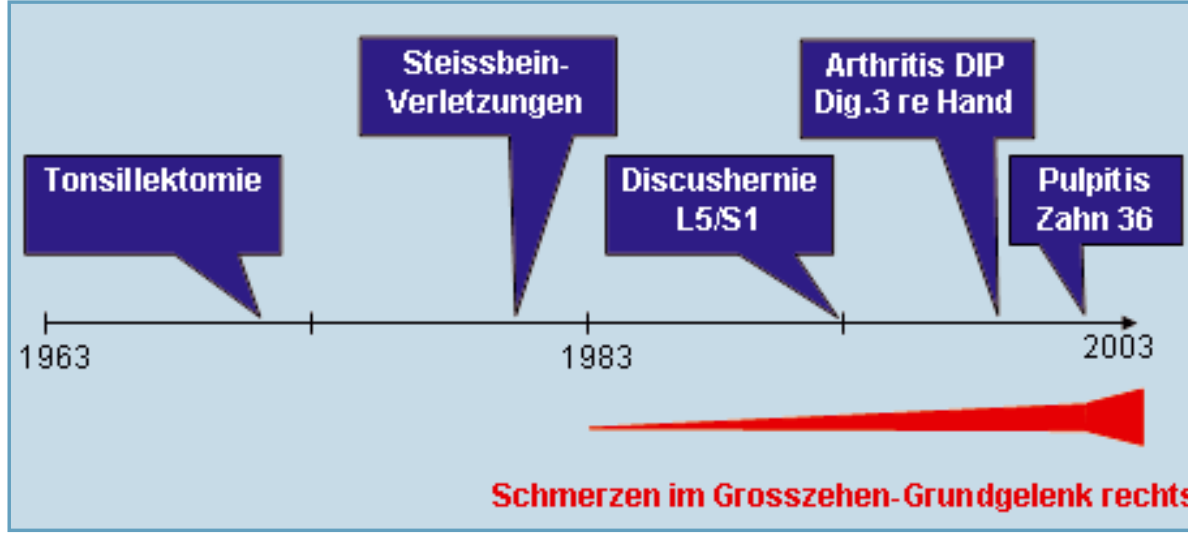

Abb. 1. Störfeldkette bei einer 40-jährigen Patientin mit Arthrose des rechten Zehengrundgelenks (Fallbeispiel 1)

einer schweren Pulpitis musste der Zahn 36 extrahiert werden. Gleichzeitig mit Beginn der Zahnbeschwerden kam es zu einer starken Zunahme von chronischen Schmerzen im Grosszehengrundgelenk des rechten Fusses. Diese Schmerzen hatten bereits 20 Jahre zuvor schleichend begonnen (Abb. 1). Vom Orthopäden wurde aufgrund der Klinik und des radiologischen Befundes eine fortgeschrittene Arthrose im Rahmen eines Hallux valgus diagnostiziert und therapeutisch eine Arthrodese vorgeschlagen. Ansonsten befand sich die Patientin in einem guten Allgemeinzustand. Die Patientin kam am 10.01.2003 mit stärksten Schmerzen in diesem Gelenk in meine Sprechstunde, sie war kaum mehr in der Lage, mehr als 100 Meter zu gehen. Das Grosszehengrundgelenk war geschwollen, allseits druckdolent und leicht gerötet. Es bestand das Bild eines akut entzündeten Hallux valgus bei normalen Harnsäurewerten im Serum.

Die Injektion von Lidocain (1\%ige Lösung) in die Extraktionsstelle des entfernten Zahnes 36 bewirkte eine vollständige Schmerzfreiheit im Bereich der Grosszehe in der Sekunde. Die Beschwerden traten erst nach 10 Tagen wieder auf.

Die Wiederholung dieser Behandlung und die zusätzliche Injektion von Procain $1 \%$ an die TonsillektomieNarben (bei St.n. Tonsillektomie in der Kindheit) führte zu einer Schmerzfreiheit von 36 Stunden. Gleichzeitig verspürte die Patientin eine Schmerzhaf- tigkeit des distalen Interphalangealgelenkes des rechten Mittelfingers. Sie erinnerte sich daraufhin, dass sie in diesem Gelenk 5 Jahre zuvor eine Arthritis unbekannter Ätiologie durchgemacht hatte. Diese als retrogrades Phänomen bezeichnete Reaktion war ein klarer Hinweis auf das Vorliegen eines zusätzlichen Störfeldes. Die Injektion mit 1\%-igem Procain in dieses Gelenk führte erneut zu einer schlagartigen Schmerzfreiheit in der rechten Grosszehe, die jedoch nur noch 30 Stunden anhielt. Gleichzeitig bemerkte die Patientin diesmal einen Schmerz lumbal mit Ausstrahlung in das rechte Bein. Sie berichtete nun, dass sie 10 Jahre zuvor eine Diskushernie L5/S1 rechts gehabt hatte, die konservativ behandelt worden war. Nach diesem erneuten retrograden Phänomen erfolgte nun eine Injektion an die Ischiaswurzel S1 rechts. Auch diesmal kam es zur vollständigen Schmerzfreiheit, aber nur noch für wenige Stunden. Ein abermals als retrogrades Phänomen zu interpretierender Schmerz im Steissbeinbereich rief der Patientin einen Sturz auf das Steissbein in der Jugendzeit in Erinnerung. Aus diesem Grund erfolgte eine sakrale epidurale Injektion sowie eine Infiltration an das Steissbein. Diese Behandlung wurde nach 5 Tagen wiederholt, worauf die Patientin schmerzfrei eine Bergwanderung unternehmen konnte. Da die Beschwerden in der Grosszehe in geringem Masse zurückkehrten, wurde diese Behandlung nach weiteren 2 und 5 Wochen wie- derholt. Seit Mai 2003 ist die Patientin völlig beschwerdefrei (Beobachtungszeit 4 Jahre). Die Entzündungszeichen (Schwellung und Rötung) im Bereich der Grosszehe rechts haben sich ebenfalls vollständig zurückgebildet.

\section{Fallbeispiel 2}

Eine 19-jährige Patientin kam wegen permanenter chronischer Kopfschmerzen in meine Sprechstunde. Diese begannen nach einer Verletzung der Kopfschwarte im Alter von 6 Jahren (der Patientin fiel ein Blumentopf aus ca. 7 Metern Höhe auf den Kopf). In den letzten 6 Monaten vor der ersten Konsultation hatte die Patientin täglich Kopfschmerzen. Diese wurden als konstant, von occipital nach frontal beidseits ausstrahlend beschrieben. Die bereits beim Erwachen bestehenden Schmerzen verstärkten sich im Laufe des Tages zunehmend. Begleiterscheinungen wie Nausea, Sehstörungen oder neurologische Ausfälle lagen nicht vor. Die Schmerzen besserten unter Analgetika (Paracetamol und nichtsteroidale Antirheumatika) nur geringfügig. Die wiederholte Infiltration der Kopfnarben mit Procain 1\% führte jeweils $\mathrm{zu}$ einer sofortigen Schmerzfreiheit von zunehmender Dauer (1; 5; 20 Tage). Nach der vierten Behandlung ging die Wirkdauer rasch zurück (6; 0 Tage), weshalb ein weiteres Störfeld vermutet wurde. Die Behandlung einer Narbe im Bereich der linken Augenbraue führte nun erneut zu einer Schmerzfreiheit von 6 Wochen, worauf dann aber sämtliche Narbenbehandlungen wirkungslos blieben. Wegen wiederholter Infekte der oberen Luftwege als Kleinkind wurden die Tonsillen neuraltherapeutisch getestet. Dabei kam es erneut zu völlig schmerzfreien Intervallen, die jedoch immer nur ca. 3-4 Wochen andauerten (insgesamt 4 Behandlungen). Die Patientin wurde daraufhin an einen HNO-Spezialisten zur Tonsillektomie überwiesen. Trotz inspektorisch weitgehend unauffälliger Tonsillen zeigte sich intraoperativ eine ausgedehnte Vereiterung beider Tonsillenlogen. Der weitere Verlauf war sehr erfreulich: nach einmaliger Nachbehandlung der 


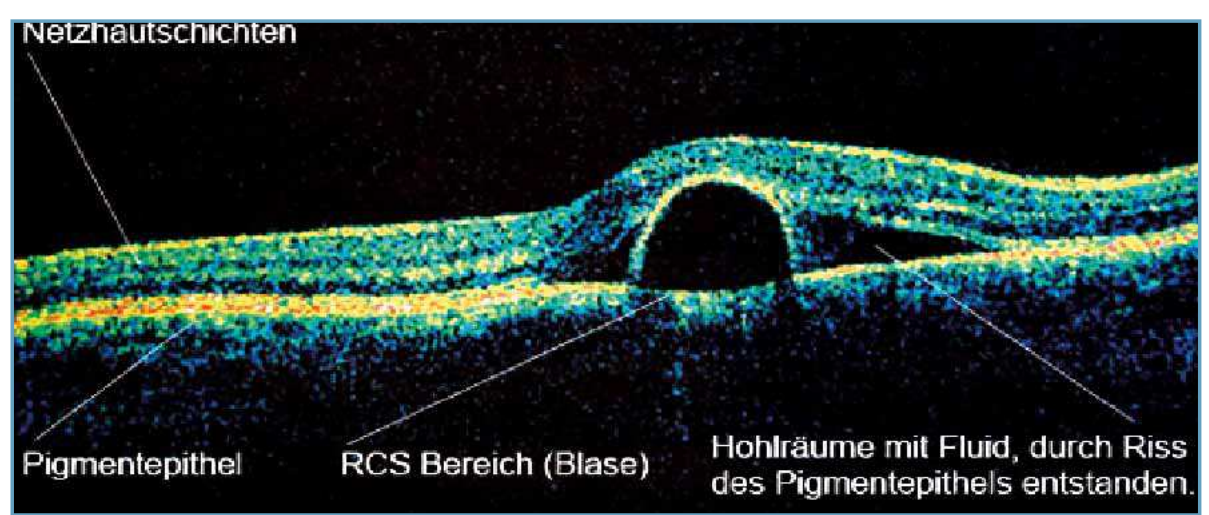

Abb. 2. Retinopathia centralis serosa RCS: Blasenbildung durch Riss im Pigmentepithel der Retina (Fallbeispiel 3)

Tonsillektomie-Narben blieb die Patientin in der Folge schmerzfrei (Beobachtungszeit > 5 Jahre).

\section{Fallbeispiel 3}

Der 41-jährige Patient litt seit 1995 unter belastungsabhängigen Leistenschmerzen beidseits. Seinen Beruf als Hochbaupolier konnte er deshalb ab dem Jahr 2003 nicht mehr ausüben. Der Patient erhielt deshalb eine 50\%ige Invalidenrente und steht zur Zeit noch in einer Umschulung. Radiologisch bestand im Bereich beider Hüftgelenke das typische Bild einer leichten bis mittelschweren Coxarthrose mit Verschmälerung des Gelenkspaltes, einer subchondralen Sklerose sowie Osteophytenbildung. Seit 13 Jahren leidet er zudem unter wöchentlichen Migräneanfällen mit Erbrechen, jedoch ohne Aura. Anfang April 2005 bemerkte der Patient einen rasch zunehmenden zentralen Sehverlust auf dem rechtern Auge. In der Augenklinik eines Universitätsspitales wurde die Diagnose einer Retinopathia centralis serosa (RCS) (Blasenbildung durch Riss im Pigmentepithel der Retina) gestellt (Abb. 2). Eine Therapie wurde vorerst nicht empfohlen.

Nach Neuraltherapie (30.06.2005) einer kleinen Narbe im Bereich des oberen Lippenbändchens infolge Sturz im Kindesalter verschwand die Sehstörung innerhalb von zwei Tagen vollständig. Ende Dezember 2005 kam es zu einem Rückfall der Sehstörung,
Lesen mit dem linken Auge war nicht mehr möglich. Das vom Ophthalmologen verordnete Diamox wollte der Patient wegen Angst vor Nebenwirkungen nicht einnehmen. Eine erneute Behandlung der Narbe im Bereich des Frenulum labii superioris war diesmal wirkungslos. Ein devitaler, wurzelbehandelter Frontzahn (Zahn 21) konnte kinesiologisch als Störfeld eruiert werden, worauf dieser am 26.02.2006 extrahiert und neuraltherapeutisch nachbehandelt wurde. Im Anschluss an diese Extraktion traten bis heute keine Migräne-Anfälle mehr auf. Zudem kam es zum sofortigen Verschwinden der Hüftgelenksbeschwerden, weshalb der Patient die IV-Rente nicht mehr beanspruchen möchte. Die Sehstörung ging subjektiv jedoch nur um ca. $70 \%$ zurück. Dies liess an ein drittes Störfeld denken. Nach nochmaliger Befragung teilte der Patient mit, dass er im Schulturnen im Bereich der rechten Grosszehe eine Distorsion erlitten hatte. Die Injektion an das Seitenband des Interphalangealgelenkes der betreffenden Zehe führte innerhalb von 2 Stunden zur Wiedererlangung der vollen Sehkraft. Die ophthalmologische Kontrolle bestätigte die Abheilung des Retina-Prozesses.

\section{Diskussion}

Bei vielen Störfeldbehandlungen erleben wir, dass nicht ein einzelnes sondern zwei bis mehrere Störfelder an einer Erkrankung beteiligt sind. Wir können in diesen Fällen von sogenannten "Störfeldketten" sprechen. In der Literatur finden sich häufig Fallberichte, in denen eine Behandlung von zwei oder gar mehreren Störfeldern beschrieben wird. Dass diese jedoch funktionell miteinander verknüpft sein können, was sich besonders durch das so genannte „retrograde Phänomen“ (symptomatisch werden eines vorher stummen Störfeldes) [3] ausdrückt, findet in der Literatur kaum Erwähnung.

Gelegentlich kann ein Störfeld auf ein erstes System einwirken, welches mit der Zeit eine eigenständige Störfeldfunktion übernehmen kann. So ist es beispielsweise möglich, dass ein impaktierter Weisheitszahn eine Störwirkung auf die Hypophyse ausübt. Diese kann sich in der Folge zu einem autonomen Störfeld entwickeln, welches z.B. eine hormonelle Dysbalance (Zyklusstörungen, Wochenbettdepression usw.) induziert. Nach Entfernung des Störfeldes Weisheitszahn [4,11] muss zur definitiven Behebung der Beschwerden anschliessend auch die Hypophyse mittels Injektion an das Rachendach $[1,2,3]$ behandelt werden.

Dass sich alle Störfelder eines $\mathrm{Pa}$ tienten in der Regel auf dasselbe Organsystem bzw. dieselbe Symptomatik auswirken, kann mit Hilfe der Biokybernetik erklärt werden. Sämtliche Störfelder bewirken durch eine permanente Reizbildung gemeinsam eine Labilisierung von Regulationssystemen. Die Symptomatik wird bestimmt durch den locus minoris resistentiae, d.h. der angeborenen oder erworbenen Schwachstelle des betreffenden Organismus.

Fallbeispiel 1 zeigt das klassische Bild einer Störfeldkette, wobei sich sämtliche Störfelder durch retrograde Phänomene diagnostizieren liessen, da diese bei der initialen Erhebung der Anamnese von der Patientin nicht angegeben worden waren. Die Tonsillektomie-Narbe war vermutlich das erste Störfeld und wurde bereits bei der zweiten Sitzung neuraltherapeutisch behandelt. Die Steissbein-Verletzung bildete den sogenannten Zweitschlag (nach dem russischen Neurophysiologen SPERANSKI, einem Schüler von PAWLOW), denn nach diesem Ereignis 
begann sich die Arthrose zu entwickeln. Die weiteren Störfelder führten zu einer zusätzlichen Belastung der Gesamtregulation, wobei die Symptomatik mit Auftreten der Pulpitis des Zahnes 36 eskalierte.

Interessant beim Therapieverlauf ist, dass sich die einzelnen Störfelder chronologisch rückwärts bemerkbar gemacht haben, ein Phänomen, das relativ häufig beobachtet wird. Es ist allerdings nicht die Regel, dass sich eine Behandlung derart klar gestaltet wie im dargestellten Beispiel. Meistens werden die einzelnen Störfelder der Kette in unterschiedlichen Abständen erneut aktiv, so dass sie wiederholt (aber auch in immer grösseren Abständen) behandelt werden müssen. Dadurch wird der Behandlungsverlauf verzögert und die Hierarchie der Störfelder ist nicht mehr so klar ersichtlich. Beherrscht der behandelnde Neuraltherapeut ein energetisches Testverfahren (z.B. Kinesiologie), mit dem er feststellen kann, welches Störfeld aktiv ist und erneut behandelt werden muss, kann dies den Therapieablauf unter Umständen erheblich vereinfachen und verkürzen.

Im Fallbeispiel 2 wurde zuerst ein Sekundenphänomen vermutet. Nach anfänglicher Verlängerung der schmerzfreien Intervalle nach Injektion in die Kopfnarben nahm die Wirkdauer plötzlich wieder ab. Dies liess ebenfalls ein übergeordnetes Störfeld ver- muten. Wenn nach wiederholter neuraltherapeutischer Behandlung eines Störfeldes (im vorliegenden Fall die Tonsillennarben) das symptomfreie Intervall mehr oder weniger konstant bleibt, spricht dies oft für einen neuraltherapeutisch nicht ausschaltbaren Herd, wie z.B. das Vorhandensein von Fremdkörpern oder, wie im vorliegenden Beispiel, eine abgekapselte, eitrige Entzündung. In diesen Fällen muss eine chirurgische Sanierung erwogen werden.

Das Fallbeispiel 3 zeigt einerseits eine typische Störfeldkette. Andererseits war hier ein Störfeld gleichzeitig für drei völlig unterschiedliche Krankheiten verantwortlich. Nach Extraktion des devitalen Zahnes 21 kam es zur völligen Beschwerdefreiheit im Bereich Hüftgelenke, die nun seit einem Jahr anhält. Ebenfalls seit dieser Zahnbehandlung traten keine Migräneanfälle mehr auf. Auch die Sehstörungen infolge der Retinopathia centralis serosa besserten sich markant. Jedoch erst nach Ausschaltung eines weiteren Störfeldes im Zehenbereich stellte sich wieder ein normaler Visus ein. Auch hier zeigt sich, dass in gewissen Fällen selbst relativ harmlose Verletzungen ohne sichtbare Folgen zur Ausbildung eines Störfeldes führen können. Oft kann in solchen Situationen nur die wiederholte, sorgfältigste Anamnese-Erhebung zum Ziel führen.

\section{Literatur}

1. Barop H. Lehrbuch und Atlas der Neuraltherapie nach Huneke. Hippokrates Verlag, Stuttgart 1996.

2. Dosch P. Lehrbuch der Neuraltherapie nach Huneke. 14. A. Haug Verlag, Heidelberg 1995

3. Fischer L. Neuraltherapie nach Huneke. Grundlagen, Technik, praktische Anwendung. 2.A. Hippokrates Verlag, Stuttgart 2001.

4. Hänisch R. Neuraltherapeutische Behandlung des Zahnstörfeldes einschliesslich dentogener Sanierung - Klinische Verläufe. Erfahrungsheilkunde 1991;40(10):678-83.

5. Heine $H$. Lehrbuch der biologischen Medizin Hippokrates Verlag, Stuttgart 2006.

6. Huneke F. Unbekannte Fernwirkung der Lokalanästhesie. Fortschr Med 1934;52(10):21323.

7. Huneke F. Neuraltherapie, Sekundenphänomen und Chirurgie. Allgemeine Therapeutik 1956;27(21):675-82.

8. Huneke F. Das Sekundenphänomen in de Heilkunde. Therapiewoche 1957:7:145-51.

9. Huneke F. Das Sekundenphänomen in der Neuraltherapie. 6. A. Haug Verlag, Heidelberg 1989.

10. Pischinger A. Das System der Grundregulation. 8. A.Haug Verlag, Heidelberg 1990

11. Stettbacher MA, Beck A. Die Bedeutung der Neuraltherapie für die Zahnmedizin. Ärztez f Naturheilverf 1991;32(9):709-15.

\section{Korrespondenzadresse:}

Dr. med. Dieter Thommen

Facharzt für Innere Medizin FMH / Neuraltherapie FMH/SANTH

Waisenhausplatz 10, CH-3011 Bern

dieter.thommen@hin.ch 